\title{
U14 base-pairs with 18S rRNA: a novel snoRNA interaction required for rRNA processing
}

\author{
Wen-Qing Liang and Maurille J. Fournier ${ }^{1}$ \\ Department of Biochemistry and Molecular Biology, and Program in Molecular and Cellular Biology, Lederle Graduate \\ Research Center, University of Massachusetts, Amherst, Massachusetts 01003 USA
}

U14 is a conserved small nucleolar RNA (snoRNA) required for processing of yeast 18S rRNA. The presence of two long sequences (13 and 14 nucleotides) with strong complementarity to $18 S$ rRNA suggests that U14 base-pairs with pre-rRNA. Evidence of direct binding was developed by showing that mutations in these U14 elements mimic U14 depletion and that function can be rescued by a compensatory sequence change in $18 \mathrm{~S}$ RNA. The U14 elements are functionally interdependent, indicating that both participate in binding. Folding models predict that binding might occur through both rRNA elements simultaneously. Potential roles of U14 in rRNA folding, maturation, and ribosome assembly are discussed. U14 is one of several snoRNAs with long complementarities to rRNA and the first snoRNA in this class shown to interact directly with rRNA.

[Key Words: snoRNA; U14; rRNA; RNA processing; ribosome synthesis]

Received July 5, 1995; revised version accepted August 23, 1995.

Several small nucleolar RNAs (snoRNAs) are known to be involved in the processing of cukaryotic rRNA (for review, see Filipowicz and Kiss 1993; Mattaj et al. 1993; Maxwell and Fournier 1995). This involvement has been demonstrated by genetic or biochemical depletion analyses, where removal of specific snoRNAs from cells or extracts has been found to disrupt processing of the large multimeric transcripts encoding $18 \mathrm{~S}, 5.8 \mathrm{~S}$, and $25 \mathrm{~S} / 28 \mathrm{~S}$ rRNAs. Processing typically begins with a cleavage in the $5^{\prime}$ external transcribed spacer segment (5' ETS), followed by cleavages at sites flanking 18S RNA (Fig. 1). The distally encoded $5.8 \mathrm{~S}$ and $25 \mathrm{~S} / 28 \mathrm{~S}$ RNAs then are released by subsequent processing reactions.

Four snoRNAs have been linked thus far to the early processing reactions (Maxwell and Fournier 1995). Two of these, U3 and U14, appear to be conserved universally, whereas the other two are currently unique to vertebrates (U22) or yeast (snR30). Each of these snoRNAs is required for processing of $18 \mathrm{~S} \mathrm{RNA}$, and $\mathrm{U} 3$ also has been tied to the 5' ETS cleavage (Kass et al. 1990; Li et al. 1990; Hughes and Ares 1991; Morrissey and Tollervey 1993; Tycowski et al. 1994). Because the depletion effects observed for these RNAs are similar, the early processing reactions are believed to take place in a complex that contains multiple small nucleolar ribonucleoproteins (snoRNPs). The functions of the snoRNAs required for processing are unknown. However, by analogy with the splicing snRNAs, they are predicted to organize the

'Corresponding author.
pre-rRNA substrates for processing and perhaps catalyze cleavage reactions as well.

U14, which is the subject of the present study, has been found in a wide range of organisms, including fungi, plants, and animals (Leader et al. 1994; Maxwell and Fournier 1995). The various homologs exhibit sequence and length heterogeneity, but each contains four conserved sequence elements and a common terminal stem (Fig. 2). These features are considered signature elements and have been useful in identifying new U14 RNAs. Two of the conserved sequences are short segments known as box $\mathrm{C}$ and box $\mathrm{D}$, which are present in many, but not all snoRNAs. Boxes $\mathrm{C}$ and $\mathrm{D}$ are required for accumulation of yeast U14 (Huang et al. 1992) and also have been implicated in binding of the nucleolar protein fibrillarin in human U3 (Baserga et al. 1991). The remaining two conserved sequences are 13- and 14-nucleotide segments complementary to different regions of $18 \mathrm{~S}$ rRNA. The presence of these elements, known as domains $\mathrm{A}$ and $\mathrm{B}$, suggests that U14 interacts directly with rRNA, through Watson-Crick base-pairing (Trinh-Rohlik and Maxwell 1988; Li et al. 1990).

The suggestion that U14 interacts with pre-rRNA is supported by mutational results with yeast U14, showing that multibase substitutions in domain $\mathrm{A}$ are lethal, whereas partial substitutions in domain B did not affect growth (Jarmolowski et al. 1990). Structural probing of yeast U14 revealed both domains to be single-stranded in protein-free snoRNA (Balakin et al. 1994). Also consistent with the possibility of base-pairing between the U14 domains and pre-rRNA is the fact that the complemen- 


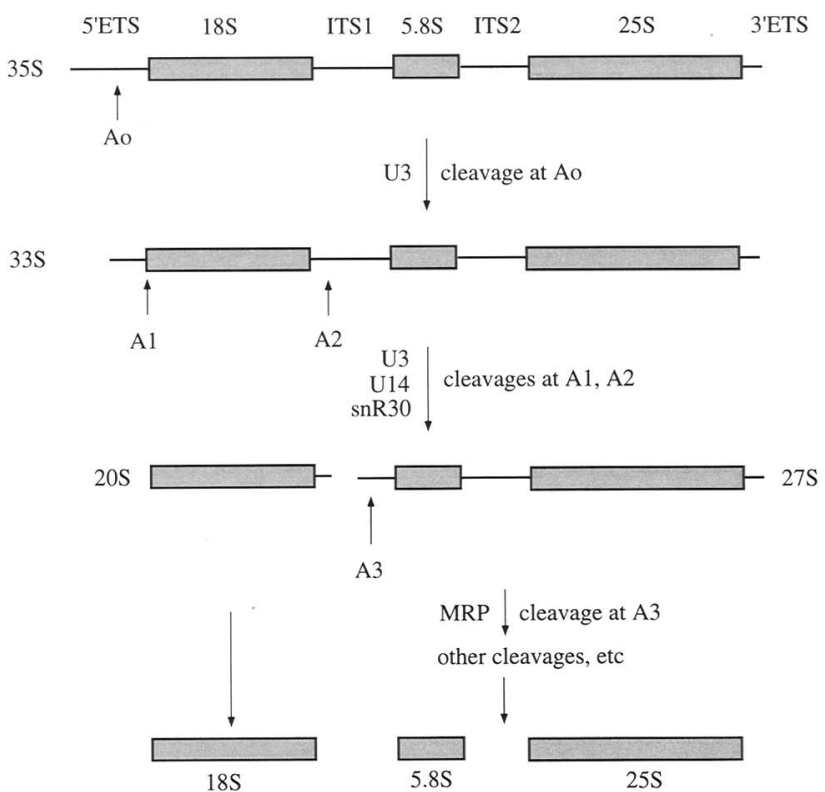

Figure 1. Processing sites in yeast pre-rRNA. 18S, 5.8S, and $25 \mathrm{~S}$ rRNAs are encoded in a $35 \mathrm{~S}$ primary transcript, flanked by the $5^{\prime}$ and $3^{\prime}$ external transcribed spacers (ETS) and separated by two internal transcribed spacer elements (ITS1 and ITS2). Processing starts at the $A_{0}$ site in the 5' ETS region, followed by cleavages at sites $A_{1}$ and $A_{2}$ that flank 18 SNA; final trimming of a $20 \mathrm{~S}$ precursor to 18S RNA occurs in the cytoplasm. U3, $\mathrm{U} 14$, and snR30 snoRNAs are required for processing at sites $A_{1}$ and $A_{2}$. U3 is also required for the $A_{0}$ cleavage. The distally encoded 5.8S and 25S RNAs are released by subsequent processing reactions. The nuclear MRP RNA is involved in cleavage at site $A_{3}$; additional cleavages are not shown. Metazoan rRNAs are processed by similar pathways.

tarities are conserved almost perfectly in all cases where both U14 and rRNA sequences can be compared. The domain A complementarities in vertebrates and yeast are nearly perfect with only one mismatch at sites in the middle portion. This is always a G:U. Potato and maize contain one or two mismatches at the $3^{\prime}$ end of domain A. The domain B complementarities are perfect in all but Saccharomyces cerevisiae and Schizosaccharomyces pombe, which contain one mismatch and one insertion, respectively. Interestingly, the complementary sequences in 18S RNA are in close proximity in the predicted secondary structure, suggesting that interaction could occur at both sites simultaneously (Fig. 3).

The present report describes genetic evidence that U14 does interact with $18 \mathrm{~S}$ rRNA through direct base-pairing and that both domain $\mathrm{A}$ and $\mathrm{B}$ are used in this interaction. We demonstrate that a lethal mutation in domain A of U14 can be rescued by a base change in 18S RNA that restores complementarity and that the domain $\mathrm{A}$ and $B$ elements are functionally interdependent. An initial effort to test the postulated binding was hampered by the high copy number of chromosomal rDNA. This complication was overcome here by using test cells containing a plasmid-borne, hygromycin-resistant rDNA allele.
Growth in hygromycin led to large losses of chromosomal rDNA, and this allowed the experimental rRNA encoded by the plasmid to dominate rRNA synthesis. This system, developed by others, provides a powerful approach for conducting genetic analysis of rRNA (Chernoff et al. 1994). In addition to showing that U14 function depends on base-pairing with IRNA, these results also provide the first genetic demonstration that a ribosomal RNA sequence participates in processing by interacting with an snoRNA.

\section{Results \\ Domain B is conditionally required for U14 function}

Evidence that the domain A sequence (13 nucleotides, with perfect complementarity to $18 \mathrm{~S}$ RNA) is important was established in the previous functional mapping stud-

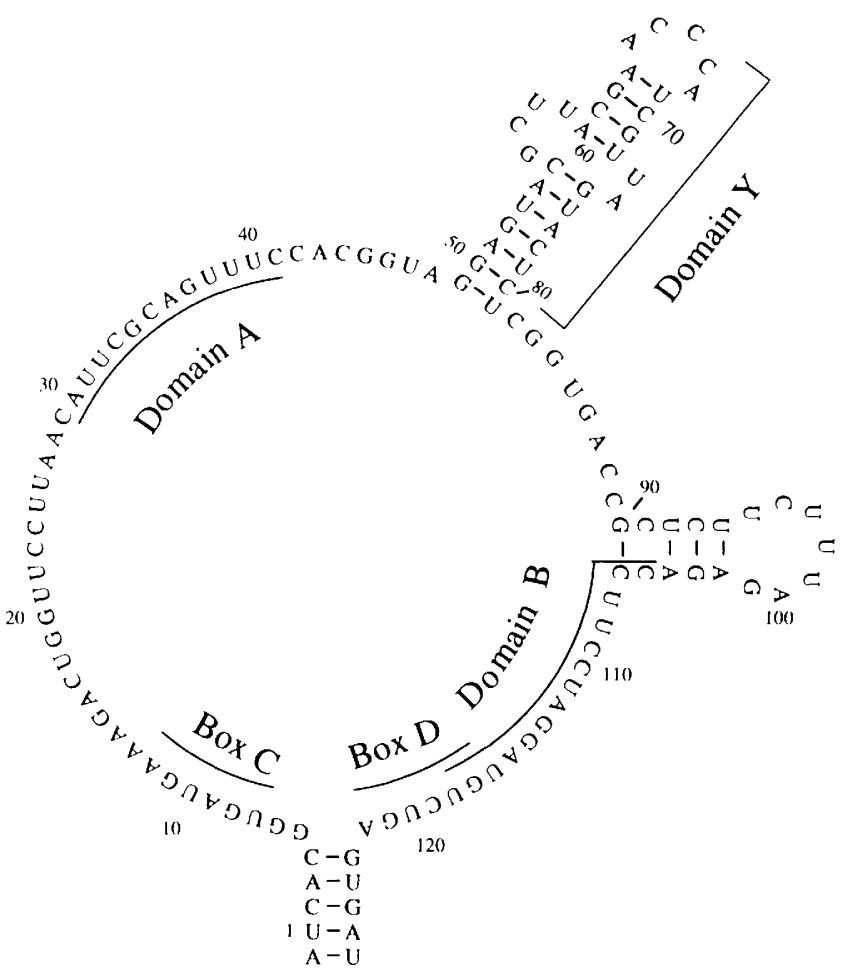

Figure 2. Structure of $S$. cerevisiae U14 snoRNA. The secondary structure was deduced from nuclease and chemical probing of $S$. cerevisiae RNA (Balakin et al. 1994). All U14 RNAs have four conserved sequence elements and a $5^{\prime}, 3^{\prime}$ terminal stem. The universal sequence elements are domains $A$ and $B$ (13 and 14 nucleotides), which are complementary to conserved sequences in 18S rRNA, and boxes C and D. Domains A and B are unique to the U14 snoRNAs, whereas boxes $C$ and D are conserved among other snoRNAs. Domain $\mathrm{Y}$ is present in U14 RNAs from yeast and plants, but not vertebrates. Functional mapping of yeast U14 has shown domains $A$ and $Y$ to be essential for activity, and boxes $C$ and $D$, and complementarity of the $5^{\prime}$ and $3^{\prime}$ ends, to be required for accumulation of U14 itself (Huang et al. 1992; Li and Fournier 1992). Domains A and B are predicted to be single-stranded in protein-free RNA. 


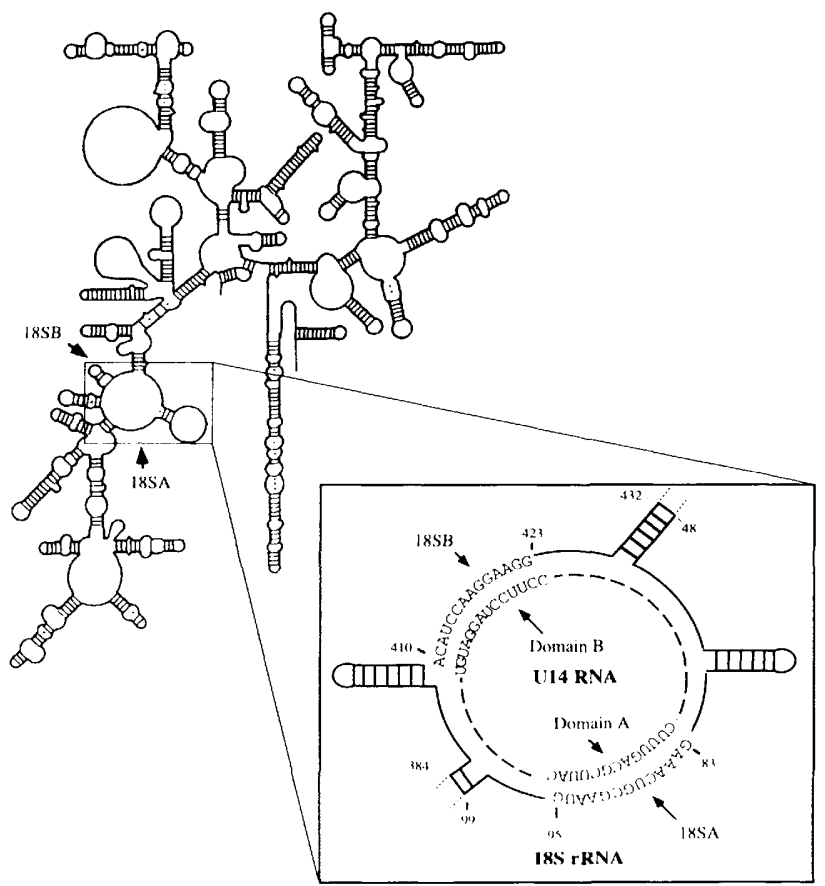

Figure 3. Potential pairing of $S$. cerevisiae Ul4 snoRNA with $18 \mathrm{~S}$ rRNA. The rRNA sequences complementary to domains $A$ and B of U14 occur in 18S RNA. These elements are predicted to be in close proximity in the secondary structure models of rRNA (Raue et al. 1988). (Inset) Hypothetical base-pairing of the complementary elements in U14 and 18S RNA. As shown, basepairing through domain $\mathrm{B}$ abolishes a stem-loop structure containing the complementary rRNA sequence. Complementarity is perfect at the domain A site, whereas a single, centrally located mismatch occurs at the domain B site. The anti-domain A sequence in pre-rRNA is 83 nucleotides from the $5^{\prime}$ end of mature 18S RNA. Loss of U14 disrupts processing at this position. The rRNA sequences complementary to U14 domains A and B are designated $18 \mathrm{SA}$ and $18 \mathrm{SB}$, respectively.

ies, where U14 was inactivated by (1) replacing either the $5^{\prime}$ or $3^{\prime}$ half of the sequence, or (2) introducing several randomly distributed base changes within domain $\mathrm{A}$ (Jarmolowski et al. 1990). The importance of domain B (14 nucleotides, one-mismatch with 18S RNA) was not clear, however, as only partial substitutions were examined and half-length elements of 7 nucleotides might be sufficient for activity. To determine whether domain B is completely dispensable, we replaced the entire element with a sequence that could not base-pair with its complementary sequence in 18S RNA. We also introduced point mutations in the middle of domain $\mathrm{A}$ (position 34 or 36 ) and examined the activity of these variants in the context of wild-type or substituted domain B sequences.

The altered U14 genes were introduced on plasmids into a test strain (YS153) that contains in the genome a wild-type U14 gene under control of the GAL1 promoter. When streaked on glucose-containing medium, U14 function is supplied by the plasmid-encoded mutant gene (Jarmolowski et al. 1990); failure to grow is indicative of a lethal U14 mutation. The results showed that substitution of the entire domain B sequence had no detectable effect on growth (Fig. 4A). Mutations at position 36 from $A \rightarrow G$ or $A \rightarrow U$ also had no effect, even in conjunction with a substituted domain B. However, a change from $G \rightarrow A$ or $G \rightarrow U$ at position 34 , in combination with replacement of domain $B$, resulted in lethality. Because the substitution of domain B was only lethal when combined with certain mutations in domain $\mathrm{A}$, we conclude that domain $B$ is conditionally dispensable. We conclude further that domains $\mathrm{A}$ and $\mathrm{B}$ are functionally interdependent, because the mutations of domain A did

$\mathbf{A}$

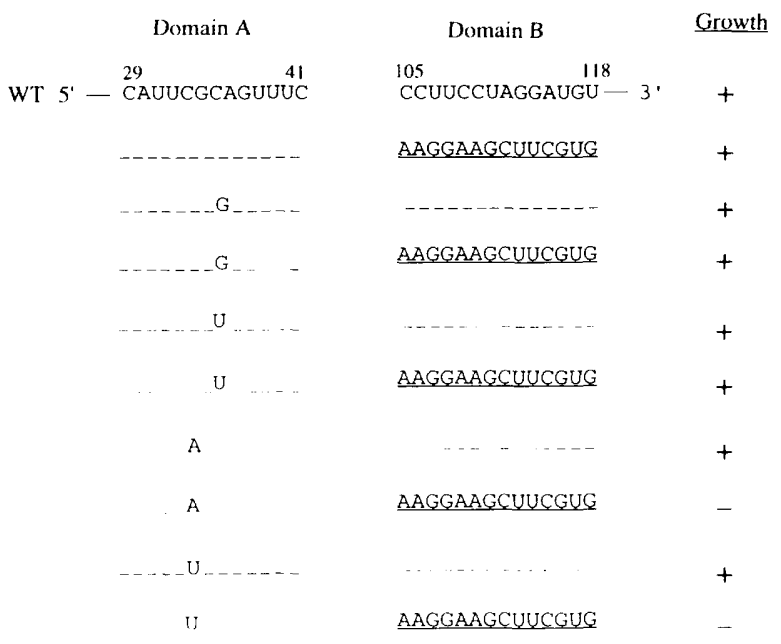

B

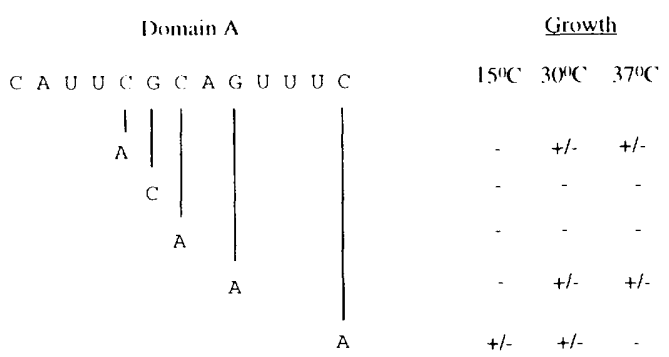

Figure 4. Effects of domain $\mathrm{A}$ and $\mathrm{B}$ mutations on growth. $(A)$ Mutations were introduced into a plasmid-encoded U14 gene and assayed for functional effects in a test strain containing a wild-type U14 gene under control of the galactose-inducible GAL1 promoter. Single-point mutations in domain A were tested with either a wild-type domain B sequence or a substituted sequence unable to pair with a complementary sequence in 18S RNA. The sequences of the mutant elements are shown beneath the wild-type $\mathrm{U} 14$ sequence. A dash indicates a wildtype nucleotide. The ability of transformants to grow at $15^{\circ} \mathrm{C}$, $30^{\circ} \mathrm{C}$, and $37^{\circ} \mathrm{C}$ on glucose medium is indicated $(+) ; 1-1$ no growth. $(B)$ Point mutations in domain $\mathrm{A}$ that cause a growth defect in conjunction with the substituted domain $B$ sequence. A pool of plasmids containing the substituted domain B sequence and mutagenized domain A elements was screened for growth defects by incubating transformants at the indicated temperatures on glucose-containing medium. Candidate mutant strains were restreaked to confirm defective phenotypes. $|-|$ No growth; $|+|-\mid$ very slow growth, compared to test cells containing a wild-type U14 gene. 
not affect growth in the context of a normal domain B, but did have an effect when domain $B$ was substituted. This interpretation is fortified by additional results presented below.

To analyze further the sequences within domain $\mathrm{A}$ that are important for function, we mutagenized domain A of a U14 gene that contained a substituted domain B, using a pool of oligonucleotides designed to create mostly single- and double-point mutations. The genes were introduced into the test strain, and after initial growth on galactose, the transformants were replica plated onto glucose medium and incubated at $15^{\circ} \mathrm{C}$, $30^{\circ} \mathrm{C}$, and $37^{\circ} \mathrm{C}$ for mutant characterization. Forty-two transformants showed growth defects at the three temperatures tested, and the plasmids contained in these isolates were shuttled into Escherichia coli and analyzed by DNA sequencing. Ten plasmids contained single mutations in domain A, 22 had double mutations, and 10 contained three or more mutations (data not shown).

Of the 10 single-point mutations identified, 5 yielded novel sequences, in addition to the G34A and G34U mutations noted above (Fig. 4B). Strikingly, all single-point mutations in either $\mathrm{G}$ or $\mathrm{C}$ positions of domain A caused growth defects. This bias was not reflected in the mutagenic oligonucleotides, as changes in $\mathrm{A}$ and $\mathrm{U}$ positions were identified among the double and triple mutations. The bias also extends to the point mutations in the previous experiment, where the mutations at A36 did not affect growth when combined with the substituted domain B sequence (Fig. 4A). In the context of domain $\mathrm{A}$ and $B$ base-pairing with complementary elements in $18 \mathrm{~S}$ rRNA, all of the mutational results can be reconciled by proposing that substitution of domain $B$ weakens the interaction of U14 with 18S RNA, and this necessitates a more stringent interaction of domain A with 18S RNA. This stringent interaction is sensitive to loss of strong $\mathrm{G}-\mathrm{C}$ base pairs, but not loss of weaker A-U interactions.

\section{Functional analysis of mutations}

in the complementary elements of $18 \mathrm{~S} r \mathrm{RNA}$

Base-pairing of the complementary elements in U14 and 18S RNA would be indicated if mutations in U14 domains A or B could be suppressed by complementary mutations in 18S RNA. However, there are two hurdles to this approach. One is the high abundance of rDNA in the chromosome, estimated at $>100$ copies (Petes and Botstein 1977). To circumvent the interference effects associated with this background, we utilized a rDNA encoding hygromycin resistance. This operon dominates rRNA synthesis when cells are grown with hygromycin (Chernoff et al. 1994).

A second difficulty in manipulating rRNA genes is the fact that the coding sequences contain information for many vital functions in addition to processing, such as base modification, ribosome assembly, and translation. A genetic approach aimed at rescuing defective domain A or B mutations with compensatory base changes in 18S RNA can succeed only if the rRNA changes have no deleterious effects by themselves. Others in our labora- tory encountered this difficulty when three different combinations of 3-base changes in the anti-domain $\mathrm{A}$ sequence of rRNA were found to block accumulation of $18 \mathrm{~S}$ RNA containing a hybridization tag. This effect was not suppressed by complementary mutations in U14 (R. Lempicki, H. Li and A. Jarmolowski, unpubl.).

In the present study we assessed the effect of compensatory base changes in 18S RNA in a yeast test strain (YS624) that lacks chromosomal rDNA and whose growth is completely dependent on a plasmid-borne GAL7-rDNA allele. Mutations in 18S RNA then could be introduced on a second plasmid and the strain transferred to glucose medium to determine whether the mutant 18S RNA can support cell growth. Because the U14G34 position appeared important for U14 function, we introduced $\mathrm{A}, \mathrm{G}$, and $\mathrm{U}$ nucleotides at the corresponding C90 position of $18 \mathrm{~S}$ RNA. Additionally, we constructed an 18S RNA gene containing the sequence complementary to the substituted domain B of U14. The mutant alleles were introduced into the test strain, along with a wild-type 18S RNA gene as a control; all test alleles were contained within an intact rDNA operon. Transformants first were grown on galactose and then streaked on glucose-containing plates at $30^{\circ} \mathrm{C}$.

We observed that cells harboring the C90U mutation grew as well as cells with wild-type 18S RNA. Thus, the C90U mutation itself was not deleterious, and this $\mathrm{mu}$ tation was suitable for our genetic analysis. In contrast, strains containing either the C90G or C90A mutations grew extremely slowly. In addition, the strain harboring the sequence complementary to the mutant U14 domain $\mathrm{B}$ (rRNA) did not grow at all on glucose medium. These deleterious mutations can be imagined to affect either the interaction of U14 and 18S rRNA or some other rRNA-dependent activity. It seems unlikely that interaction with U14 is impaired, as U14 alleles with mutations at position G34 or substitution of domain B should have been affected in the same way, yet were still functional. For this reason we believe it is more likely that the defects reflect loss of some function other than U14 binding.

\section{The U14-G34A mutant can be suppressed by a $18 S-C 90 U$ mutation}

Having demonstrated that the 18S-C90U mutation does not block any essential rRNA-dependent process, we coupled this mutation with the hygromycin-resistant mutation in a plasmid-borne rDNA operon. This plasmid was then transformed into a galactose-dependent U14 test strain (YS153), along with various U14 gene variants on a separate plasmid (Fig. 5). In the presence of hygromycin and glucose, transformants derived their U14 RNA and most of their 18S RNA from the plasmidencoded genes (see below). Thus, this strain permits analysis of compensatory mutations introduced into both 18S RNA and U14 genes.

Strains containing the plasmid-encoded 18S RNA were used to test U14 genes containing either the wildtype domain A sequence or alleles with the C35A mu- 


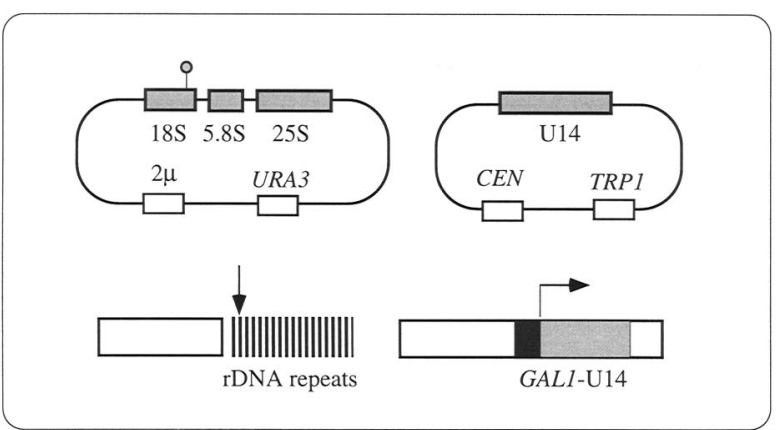

Figure 5. Genetic system for testing the proposed base-pairing of U14 with $18 \mathrm{~S}$ rRNA. The test strain contains a GAL1-U14 gene in the chromosome and a mutant U14 allele on a single copy $(C E N)$ plasmid. Growth on glucose medium depends on active plasmid-encoded U14 RNA. In addition, the strain contains a plasmid-borne rDNA operon. This operon is under the control of its cognate promoter and contains a mutation in the 18 S RNA-coding sequence that confers resistance to hygromycin. When grown on medium containing hygromycin B, most of the 18S rRNA expressed is derived from the plasmid-borne operon (see Fig. 7), because of large deletions of the chromosomal rDNA (Chernoff et al. 1994). The structures of the plasmids used are depicted at the top; the relevant chromosomal alleles are shown at the bottom. The rDNA allele encoding hygromycin resistance is marked (upper left). The vertical arrow depicts loss of chromosomal rDNA. The postulated base-pairing of U14 and $18 \mathrm{~S}$ rRNA was examined by introducing mutations in the plasmid rDNA and U14 alleles.

tation or mutations at G34. These variants contained either a wild-type or substituted domain B element. Transformants were grown first for 5 days on plates containing hygromycin plus galactose, then streaked on plates containing hygromycin plus glucose for growth analysis at $15^{\circ} \mathrm{C}, 30^{\circ} \mathrm{C}$, and $37^{\circ} \mathrm{C}$.

As expected, all Ul4 mutants could grow on medium containing both hygromycin and galactose because of the induced expression of the chromosomal U14 gene (data not shown; Jarmolowski et al. 1990). Additionally, the presence of the C90U mutation in the plasmid-encoded 18S rDNA was not detrimental to strains producing either wild-type U14 or U14 with a substituted domain B element (Fig. 6, A-D). As observed previously, the U14-G34A mutation did not affect growth when the normal domain B sequence was present, but was lethal in conjunction with the substituted domain B sequence (Fig. 6E,F). Mutation of U14-G34 to U or C also caused lethality in combination with the substituted domain $B$ sequence (Fig. 6K, M), as did the mutation C35A (Fig. $6 \mathrm{H})$. The $18 \mathrm{~S}-\mathrm{C} 90 \mathrm{U}$ mutation suppressed the mutant phenotype of the domain B-substituted U14-G34A allele, allowing growth on glucose at all three temperatures tested (Fig. 6G). The C90U change in 18S rRNA could not, however, suppress the U14-G34U, -G34C, or -C35A mutations (Fig. 6L,N,I). Because only A-U rather than $\mathrm{C}-\mathrm{U}$ or $\mathrm{U}-\mathrm{U}$ pairing can restore growth, the observed suppression is base specific. The requirement for base complementarity is strong evidence for direct basepairing of the U14 and 18S RNA elements.

\section{Analysis of $18 S$ rRNA and U14 expressed in cells grown with hygromycin}

To examine expression of 18S rRNA within the various transformants, we performed a primer extension termination assay, which permits $18 \mathrm{~S}$ RNA containing C90U to be distinguished from wild-type RNA, by virtue of a longer termination product (Fig. 7A; Sigmund et al. 1988). In transformants containing the mutant 18SC90U gene on a plasmid, most of the extension products were derived from this allele when cells were grown in the presence of hygromycin. In the absence of antibiotic the wild-type C90 product was dominant (Fig. 7B, cf. 2 and 81. These results confirm that introduction of the hygromycin-resistant mutation and subsequent growth on hygromycin results in predominant expression of plasmid-encoded rDNA, rather than chromosomal rDNA.

The 18S-C90U RNA is expressed strongly in all strains grown on either glucose or galactose media containing hygromycin; the relative amount of plasmid-derived 18S RNA produced in the presence of hygromycin is at least as much as that derived from the chromosome in the absence of this antibiotic. Only a small amount of $18 \mathrm{~S}-\mathrm{C} 90 \mathrm{U}$ rRNA is expressed in the absence of hygromycin (lane 2). This may explain why the U14-G34A/ substituted domain B mutant phenotype could not be rescued fully in transformants containing the compensatory 18 S RNA mutation when grown on glucose without hygromycin.

Expression of U14 was examined by Northern analysis (Fig. 8), where the GAL1-regulated chromosomal U14 gene produces a slightly longer product than the plasmid-borne U14 alleles ( $\mathrm{Li}$ and Fournier 1992). None of the strains grown on glucose showed any GAL1-derived U14 RNA, confirming that the base-specific suppression observed between U14-G34A and 18S-C90U is not attributable to leaky expression of wild-type, chromosomally encoded U14. Northern analysis also confirmed that the U14-G34C allele was expressed well in strains containing the 18S-C90U mutation (Fig. 8, lane 9). Because the previous experiment demonstrated that U90 rRNA was found in cells containing this U14 allele (Fig. 7B, lane 10), we conclude that the growth defect of the strain containing U14-G34C and 18S-C90U rRNA is attributable to the failure of base-pairing between these two RNA species.

As an additional control, all of the test sequences were determined to be unchanged by shuttling the plasmids from the yeast transformants into $E$. coli and sequencing the U14 genes and 18S rDNA region of interest. This eliminated the possibility that suppression resulted from simple reversion or bypass of the original mutations.

Suppression of U14-G34A by a compensatory mutation in $18 S$ RNA restores the steady-state level of $18 S$ rRNA

To determine whether suppression by $18 \mathrm{~S}-\mathrm{C} 90 \mathrm{U}$ can restore the steady-state level of $18 \mathrm{~S}$ rRNA in the U14G34A mutant, we isolated RNA from cells that had been 
A.

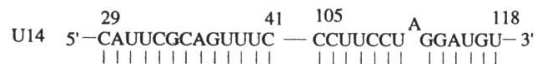

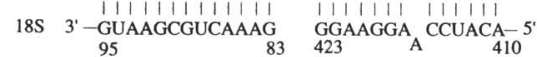

B.

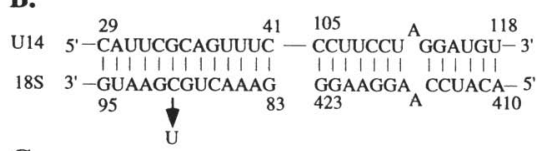

C.

$$
\begin{aligned}
& \text { U14 } \stackrel{29}{29} \stackrel{41}{105} \stackrel{118}{105} \\
& \begin{array}{l}
185 \text { 3'-GUAAGCGUCAAAG } \\
\text { 95 }
\end{array} \\
& \begin{array}{l}
\text { GGAAGGAACCUACA- } 5^{\prime} \\
423
\end{array}
\end{aligned}
$$

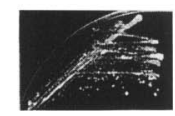

D.

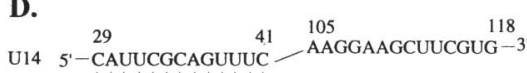

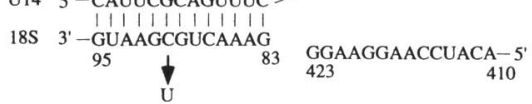

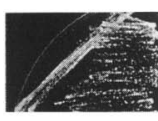

Figure 6. Effects of $18 \mathrm{~S}$ rRNA mutations on growth of strains containing mutant U14 alleles. The sequences of U14 domains $\mathrm{A}$ and $\mathrm{B}$ are shown with the complementary 18S rRNA sequences. The wild-type sequences are shown in $A$. Mutations introduced into domains $\mathrm{A}$ and the complementary sequence in rRNA are indicated with arrows. Base-pairing between domain $\mathrm{B}$ and $18 \mathrm{~S}$ RNA is disrupted in the strains shown in C-D, F-I, and $K-N$, due to a substituted domain B in U14 RNA. Growth phenotypes were analyzed by streaking strains containing the indicated U14 and 18S RNA gene combinations onto glucose medium and incubating at $15^{\circ} \mathrm{C}, 30^{\circ} \mathrm{C}$, and $37^{\circ} \mathrm{C}$. The growth patterns shown are for $37^{\circ} \mathrm{C}$.

shifted to glucose medium for $40 \mathrm{hr}$ and analyzed the rRNA pattern by gel electrophoresis and ethidium bromide staining (Fig. 9). RNA from galactose-grown cells served as a control. Regardless of whether the plasmidborne 18S RNA allele carried the C90U mutation or not, all transformants produced similar amounts of 18S RNA when grown on galactose, with no apparent difference in the ratio of $18 \mathrm{~S}$ and $25 \mathrm{~S}$ RNAs (lanes 2,4,6). Transformants containing the wild-type, plasmid-encoded $18 \mathrm{~S}$ rDNA and U14 genes also produced normal amounts of I8S RNA when shifted to glucose-containing medium (lane 1).

However, transformants containing the wild-type $18 \mathrm{~S}$ RNA allele along with the U14-G34A mutant gene showed a deficiency in 18S rRNA /compared to $25 \mathrm{~S}$ RNA) when grown on glucose-containing medium (lane 3). Jarmolowski et al. (1990) observed a similar underaccumulation of 18S RNA in strains dependent on a U14 gene that contained other domain A mutations. In contrast, normal levels of $18 \mathrm{~S}$ rRNA were found to accumulate in cells containing both the defective U14-G34A mutation and the suppressing 18S-C90U mutation (lane 5). This finding demonstrates that restoring complementarity between U14-G34A and 18S-C90U rRNA results in normal production of $18 \mathrm{~S}$ rRNA. Taken together, the results show that the U14-G34A mutant phenotype can be suppressed in nucleotide-specific fashion by a com-

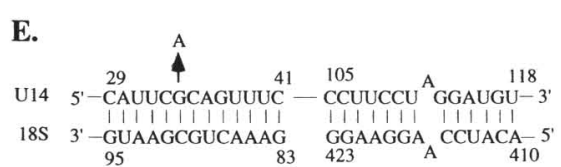

Growth
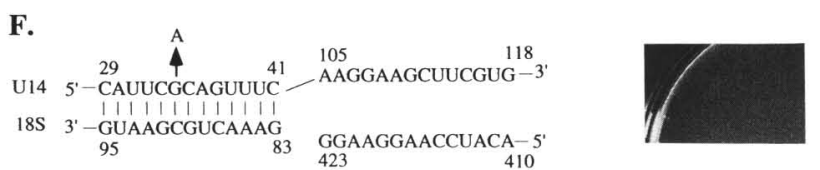

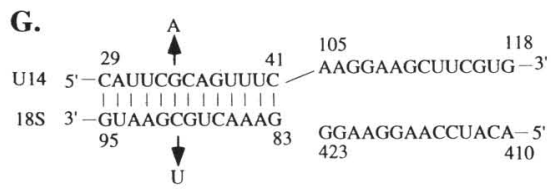

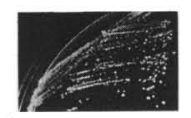

H.
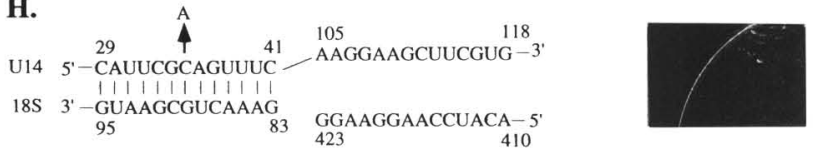

I.
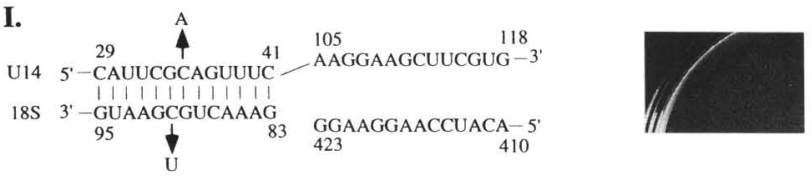

J.

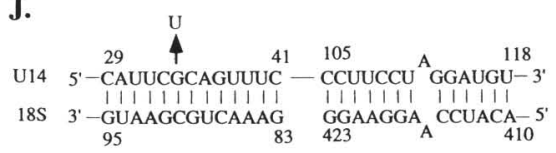

Growth
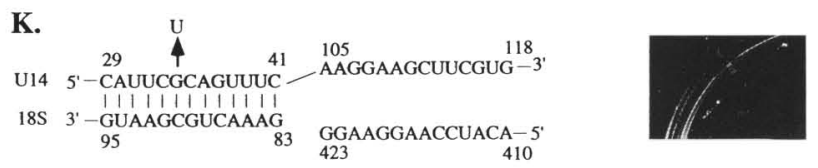

L.
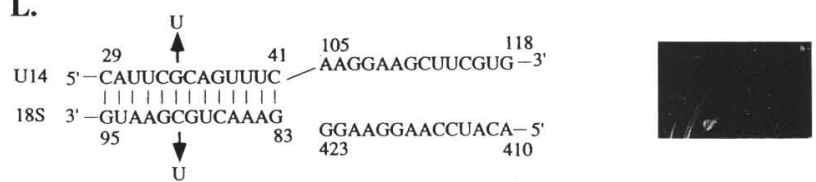

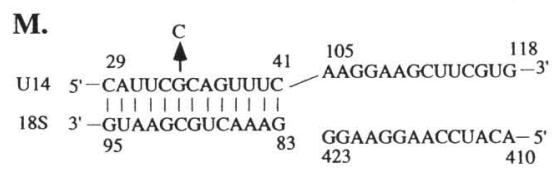

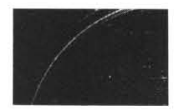

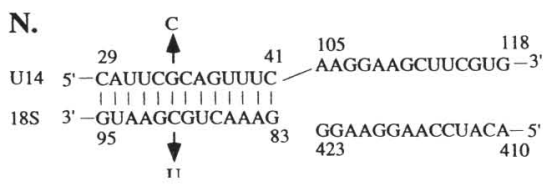

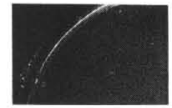

pensatory change in 18S rRNA and that this suppression restores the normal steady-state level of $18 \mathrm{~S}$ rRNA.

\section{Discussion}

Our results show that (1) the domain A and B elements conserved among all U14 RNAs are essential for U14 activity, and (2) U14 interacts directly with the complementary sequences in 18S RNA, at least through domain A and most likely through domain B as well. U14 func- 
$\mathbf{A}$

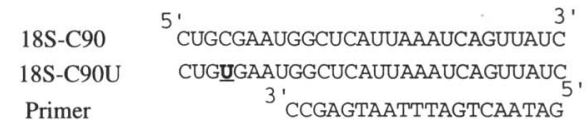

ddG termination products

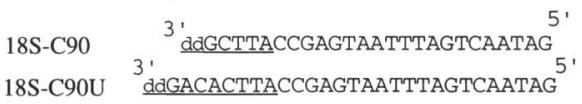

B

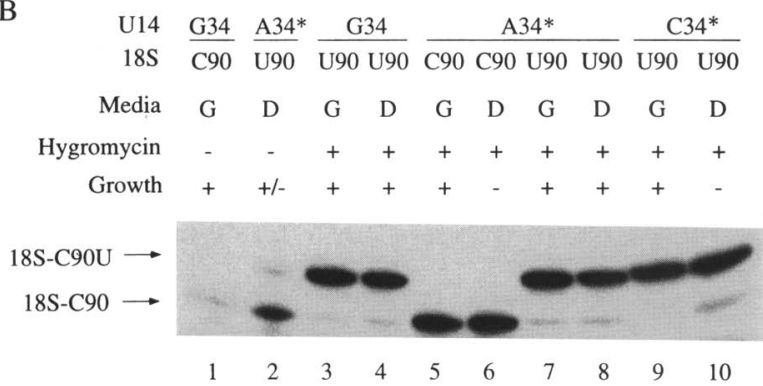

Figure 7. Expression of plasmid-encoded 18S rRNA. (A) A dideoxy primer extension termination assay was used to discriminate between 18 S RNA derived from the plasmid and chromosomal rDNA operons. The hygromycin-resistant C90U $18 \mathrm{~S}$ rRNA could be distinguished from wild-type 18S RNA, as basespecific termination produced a 28 - vs. 26-nucleotide product cDNA. $(B)$ Total RNA from cells containing the various mutant U14 and $18 \mathrm{~S}$ alleles in Fig. 6 was reverse-transcribed with a primer complementary to nucleotides $96-116$ of 18 S rRNA. The extension reaction was carried out with reverse transcriptase in the presence of dideoxy-GTP. The plasmid-borne U14 genes contained either the wild-type G34 allele or the G34C or G34A mutations in conjunction with a wild-type or substituted domain $B$ (the latter is indicated by an asterisk). The plasmidborne 18S RNA genes contained either the wild-type C90 allele or the C90U mutation that is complementary to U14-G34A. All 18S rDNA alleles contained the hygromycin-resistant mutation. RNA was prepared from transformants grown either on galactose $(G)$ or glucose (D; after initial growth on galactose) with or without hygromycin. Equal amounts of radioactivity were loaded, except for lane 1 , which is a wild-type control. $(+1$ Wild-type growth; $(-)$ a lethal phenotype. The arrows at left identify the rDNA transcripts from the hygromycin-resistant test allele (C90U, plasmid) and chromosomal rDNA (C90).

tion was retained when domain $\mathrm{B}$ was replaced with a sequence that cannot base pair with a complementary sequence in $18 \mathrm{~S}$ RNA. Function was lost, however, on combining the substituted domain B mutation with several point mutations in domain $\mathrm{A}$, indicating that domains $A$ and $B$ are functionally interdependent.

Evidence of direct base-pairing between U14 and the complementary sequences in 18S RNA came from demonstrating that growth of a mutant U14 allele can be restored through a compensatory base change in the antidomain A element of 18S rRNA. Specifically, U14 with a G34A mutation and substituted domain B could be suppressed by a C90U mutation in $18 \mathrm{~S}$ RNA, whereas

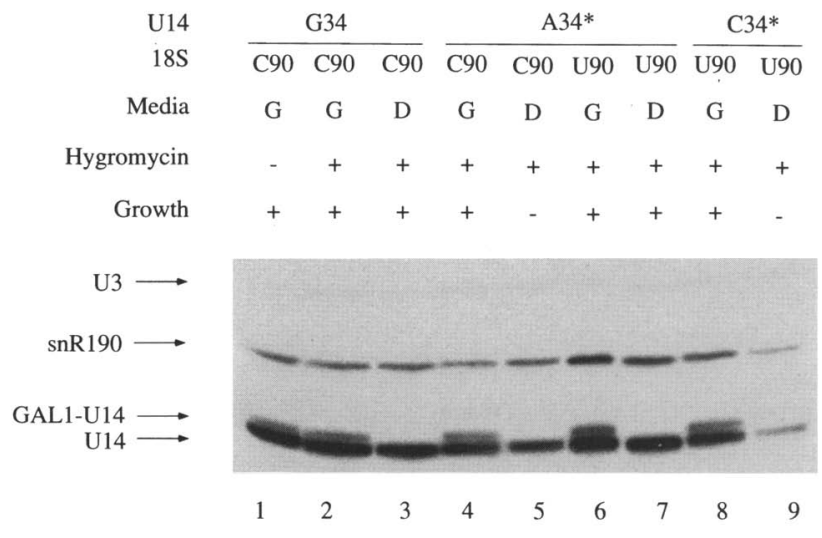

Figure 8. U14 RNA levels in the test cells. The relative abundance of mutant and wild-type U14 RNAs from the cells depicted in Fig. 7 was determined by Northern analysis. Total RNA was fractionated in a $10 \%$ denaturing polyacrylamide gel and the blot probed with a ${ }^{32} \mathrm{P}$-labeled oligonucleotide specific for U14. U14 produced from the chromosomally encoded GAL1 allele contains five extra nucleotides at the $5^{\prime}$ end and can thus be distinguished from plasmid-encoded U14 RNA. Sample loading was assessed with probes for U3 (low specific activity) and snR 190 (lane 9 is underloaded). The experimental U14 and 18S RNA genes, media, and growth phenotypes are described in Fig. 7.

lethal alleles U14-G34C and G34U or C35A, all with substituted domain B elements, could not be suppressed by this alteration in $18 \mathrm{~S}$. This base-specific suppression argues that the $18 \mathrm{~S}$ rRNA precursor must base-pair with U14 to allow processing.

All of the single-point mutations in domain $A$ that caused lethality in combination with the substituted do-

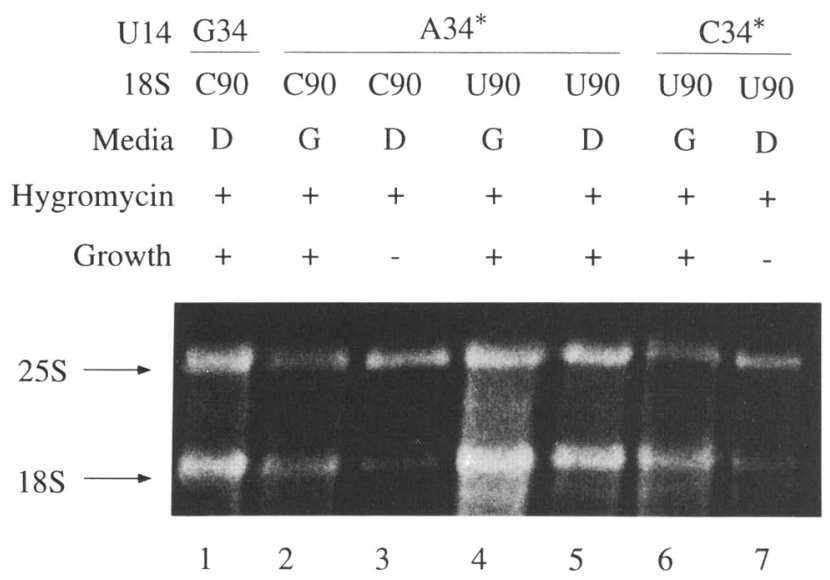

Figure 9. Steady-state levels of rRNA. Total RNA complexed with ethidium bromide was fractionated on a $1.2 \%$ agaroseformaldehyde gel. The $18 \mathrm{~S}$ and $25 \mathrm{~S}$ rRNA species were visualized under a UV light and the relative amounts of 18S RNA and 25S RNA compared for each sample. Quantitation showed the ratios for fully complementary U14:18S RNA pairs to be near normal, whereas a marked deficiency in 18S RNA occurred where complementarity was impaired (lanes 3,7$)$. The experimental U14 and 18S RNA genes, media, and growth phenotypes are described in Fig. 7. 
main $B$ resided in $\mathrm{G}$ or $\mathrm{C}$ positions. This is taken as evidence that if the domain $\mathrm{B}$ interaction is eliminated, the $13 \mathrm{bp}$ that can form between domain $\mathrm{A}$ and its complement in 18S RNA becomes critical for function and that disruption of strong $G-C$ pairs can cause lethality.

The success of this analysis relied on overcoming the high abundance of chromosomally encoded wild-type rRNA and the multifunctional nature of the rRNA segments of interest. The first challenge was met by coupling the compensatory mutations in 18S RNA with a mutation that causes hygromycin resistance, in a plasmid-borne rDNA allele. Expression of the endogenous rDNA was reduced dramatically, and that of the plasmid rDNA was enhanced dramatically, by culturing with hygromycin; this condition has been shown to lead to massive deletions of chromosomal rDNA /Chernoff et al. 1994). This powerful genetic system should be well suited for testing interactions of other snoRNAs with rRNA, as well as mapping other vital rDNA elements.

The second challenge was overcome by developing a strain containing no detectable endogenous rDNA and whose $18 \mathrm{~S}$ rRNA was derived solely from a $G A L 7$-regulated plasmid-borne rDNA operon. Using this strain we showed that the 18S-C90U mutation was not deleterious to growth, arguing that this mutation does not affect other 18S RNA-dependent activities. However, other potential compensatory mutations did not permit growth in this assay, rendering them unsuitable for our suppression analysis.

Although we have demonstrated direct interaction between U14 domain A and the complementary region of 18S RNA at positions 34 and 90 , respectively, it is not clear if the sites of interaction between domain $A$ and rRNA are limited to the sequence examined. An especially intriguing candidate occurs 8 nucleotides from the 3 ' end of mature $18 \mathrm{~S}$ RNA. This segment contains only two mismatches with domain $A$, one of which is a $G: U$ base pair. Several much shorter regions of complementarity also exist between domain A and rRNA, and the possibility that domain A might interact with other rRNA segments cannot be ruled out. No other long complementarities to domain B exist in rRNA.

The pre-rRNA processing pathway in yeast has been well defined (Woolford and Warner 1991; Eichler and Craig 1994; Henry et al. 1994; Raue and Planta 1995|. It is generally accepted that the $35 \mathrm{~S}$ primary transcript is first cleaved at a site in the $5^{\prime}$ external transcribed spacer known as $\mathrm{A}_{\mathrm{O}}$ (Fig. 1). This cleavage might be equivalent to the initial 5' ETS cleavage known to occur in vertebrate pre-rRNAs, but this has not been established. The $A_{0}$ cleavage is normally followed by cleavages at the $A_{1}$ and $\mathrm{A}_{2}$ sites, which create $20 \mathrm{~S}$ and $27 \mathrm{~S}$ intermediates. The $20 \mathrm{~S}$ precursor eventually is converted to 18S RNA in the cytoplasm, whereas the $27 \mathrm{~S}$ precursors undergo further cleavages in the nucleolus to produce mature $25 \mathrm{~S}$ and 5.8S RNAs. As noted earlier, U14, U3, and snR30 are all essential for processing of 18S RNA.

A step that occurs very early in the pre-rRNA processing pathway is the formation of a stable complex in the $5^{\prime}$ ETS segment. These complexes have been visualized cytologically and correspond to the terminal balls in Miller Christmas tree spreads of Xenopus rDNA transcription complexes (Mougey et al. 1993b). Complexes believed to correspond to these structures also have been detected biochemically, by electrophoretic analysis of both mouse and Xenopus cell extracts incubated with in vitro rDNA transcripts (Kass and Sollner-Webb 1990; Mougey et al. 1993a). Failure to assemble this primary processing complex abolished the $5^{\prime}$ ETS cleavage in mouse and Xenopus. U3, which is required for the $5^{\prime}$ ETS cleavage in mouse, Xenopus, and yeast, is believed to be part of this complex (Kass et al. 1990; Hughes and Ares 1991; Mougey et al. 1993a) and to interact directly with the 5 ' ETS region. U3 has been cross-linked to the 5' ETS segment in human, rat, mouse, and yeast, although to different sites (Maser and Calvet 1989; Stroke and Weiner 1989; Beltrame and Tollervey 1992; Tyc and Steitz 1992). Cross-linking of yeast U3 occurs through a complementary sequence of 10 nucleotides, and this complementarity has been shown to be essential for U3 activity. Direct base-pairing has been implicated, based on rDNA deletion studies and, most compelling, on rescue of activity through compensatory base changes (M. Beltrame and D. Tollervey, pers. comm.). In addition to $\mathrm{U} 3$ and U14, yeast snR30 is also believed to interact with rRNA, based on chemical cross-linking (Morrissey and Tollervey 1993).

It is reasonable to propose that U14 may be a component of the initial processing complex, at least in vertebrates. Recently, U14 also has been found to be required for the 5' ETS cleavage event in mouse extracts, using a short pre-rRNA substrate /C. Enright and B. SollnerWebb, pers. comm.). This rRNA substrate lacks the $18 \mathrm{~S}$ RNA elements complementary to U14, indicating that U14 binding to 18S RNA is not required for the ETS cleavage. In yeast, Ul4 is not required for this first cleavage, as depletion has no observable effect on processing at the $A_{0}$ site but, instead, inhibits cleavages at $A_{1}$ and $\mathrm{A}_{2}$ (Li et al. 1990; Beltrame et al. 1994).

The roles of the snoRNAs that influence the $A_{1}$ and $A_{2}$ cleavages in yeast are less clear. One attractive hypothesis is that U3, U14, and perhaps other snoRNAs such as snR30, may form the core of a "processome" complex that initiates and facilitates pre-rRNA processing through snoRNA-rRNA interactions, analogous to the splicing snRNAs in mRNA maturation. In one model, eukaryotic processing is predicted to proceed in a fashion similar to that in prokaryotes, with snoRNAs bringing together sequences that flank the rRNA segments (Morrissey and Tollervey 1995), followed by stepwise cleavages that include an RNase P-like activity [the mitochondrial RNA processing (MRP) snoRNA; Schmitt and Clayton 1993; Chu et al. 1994; Lygerou et al. 1994]. The early-acting snoRNAs, functioning as snoRNP complexes, can be imagined to mediate many of the activities involved in transcript maturation, including prerRNA folding, base modification (see below), assembly of ribosomal ribonucleoprotein (RNP) complexes and actual hydrolysis. The snoRNAs that bind directly to prerRNA are obvious candidates for roles in substrate re- 
modeling or hydrolysis. In the context of the present study, we are attracted to the possibility that U14 influences rRNA folding or rRNP assembly reactions, perhaps as an RNA chaperone. In this capacity, negative effects associated with competing, nonproductive reactions could be minimized by the U14 snoRNP. Binding and release of U14 could also serve as a checkpoint in monitoring ribosome assembly.

U14 was the first member of a growing class of snoRNAs with long complementarities to rRNA. Ten species have now been identified that contain complementary elements of 13 or more nucleotides (for review, see Bachellerie et al. 1995). U14 is the first of these to be shown to interact with rRNA through these complementary sequences. Evidence that the others might do so as well includes the fact that the complementary elements are phylogenetically conserved. Interestingly, all of the complementarities in rRNA occur in the conserved inner core region.

In addition to serving as effectors or modulators of RNA folding or subunit assembly, the complementary snoRNAs also have been postulated to have potential roles in base modification of rRNA (Bachellerie et al. 1995). This hypothesis is based on the observation that the complementary rRNA segments correlate with sites of base modification and are enriched in modified bases. This includes the anti-domain B element of $18 \mathrm{~S}$ rRNA, which, in vertebrates and in a yeast closely related to $S$. cerevisiae (Saccharomyces carlsbergensis), contains two 2 '-O-ribose-methylated nucleotides (Raue et al. 1988). In principle, U14 could provide sequence specificity for one or more modifying enzymes or play a role in forming an active substrate. In the context of processing, the defects observed could reflect loss of function related directly or indirectly to base modification. Examining the effects of U14 loss on base modification and rRNP assembly will be important aims for future studies.

\section{Materials and methods}

\section{Strains and media}

Yeast strain YS153 is MAT $\alpha$, ura3, trp1, his3, HIS3::GAL1:: SNR128 (snR128 is U14). Wild-type U14 in this strain is expressed from a chromosomally encoded galactose-inducible allele (Jarmolowski et al. 1990). YS625 is MATa, ade2, trp1, ura3, leu2, his3. Cells were cultured in medium containing $0.67 \%$ yeast nitrogen base (amino acid-free), $2 \%$ vitamin-free casamino acids, and $2 \%$ glucose or galactose, supplemented with uracil $(200 \mu \mathrm{M})$ or tryptophan $(400 \mu \mathrm{m})$ as needed. Cells and cultures were manipulated as described by Sherman et al. (1985). Hygromycin B was purchased from Calbiochem and used at a concentration of $0.5 \mathrm{mg} / \mathrm{ml}$ following filter sterilization.

\section{rDNA subcloning and mutagenesis}

DNA manipulations were carried out with standard procedures (Ausubel et al. 1989; Sambrook et al. 1989). Plasmid pJZ45 was used for U14 mutagenesis (Jarmolowski et al. 1990). It contains a 1.3-kb yeast genomic fragment encoding the snR 190 and U14 snoRNAs, a TRP-ARS for plasmid selection, and a CEN3 element for low-copy control. The mutagenesis procedure was after Kunkel (1985). Single-stranded template was purified and used in a DNA synthesis reaction according to a protocol provided by Bio-Rad, using helper phage R408 from Stratagene. Plasmid YEp24-rDNA consists of a YEp24 plasmid with a rDNA operon derived by joining two HindIII fragments and insertion at BamHI and Sall sites. A variant of this plasmid, YEp24-t-rDNA, contained a 24-nucleotide hybridization tag in variable region 2 of the $18 \mathrm{~S}$ RNA-coding sequence (Dams et al. $1988 ; \mathrm{Li} \mathrm{1991)}$. The tag sequence is 5'-AGCCCCCTAGTTGGATCCTCGGCG-3' and contains a BamHI site. The wild-type rDNA fragments were provided by J. Warner (Elion and Warner 1984). A $0.8-\mathrm{kb}$ fragment of $18 \mathrm{~S}$ rDNA produced by HindIII and SacIl and a 6.4-kb BamHI fragment containing the downstream portion of the large rDNA operon were subcloned for mutagenesis into the $\mathrm{pBluescript}$ (SK + | vector from Stratagene. Mutated rDNA fragments were subcloned back into the YEp24-t-rDNA plasmid after sequencing. The hygromycin-resistant mutation was created at the $3^{\prime}$ end of $18 \mathrm{~S}$ RNA according to Chernoff et al. (1994) and Spangler and Blackburn (1985). Plasmid pWL53 is a LEU2 high copy plasmid (YEp366) containing a 0.5-kb 5S RNA gene fragment, prepared by PCR amplification. The primers (with HindIII tails) were $5^{\prime}$-GTTTGTCCGAATCGAGTG-3' and 5'-CAACTTTCATGTTCTGTTTCG-3'. A GAL7-rDNA fragment was subcloned into this plasmid at BamHI and SalI sites, to yield plasmid pWL55. The latter rDNA fragment was derived from pNOY199 provided by M. Nomura (Nogi et al. 1991).

\section{Construction of a galactose-dependent rDNA test strain}

The high-copy plasmid YEp24-rDNA containing a hygromycinresistance mutation was transformed into yeast strain YS625, and a strain lacking chromosomal rDNA (YS626) was isolated as described by Chernoff et al. (1994). Strain YS626 was transformed with pWL55, a LEU2 high-copy plasmid containing both a GAL7-rDNA allele and a 5S RNA gene. The transformants subsequently were grown on galactose medium containing 5-fluoro-orotic acid (5-FOA) to cure the cells of the YEp24rDNA $\left(\mathrm{Hyg}_{\mathrm{R}}\right)$ plasmid. The surviving cells were screened for growth on glucose medium and an isolate able to grow on galactose, but not glucose, was chosen (YS624).

\section{RNA analyses}

RNA was extracted from cells at densities of $0.5-1.5 \mathrm{OD}_{600}$. Cells were resuspended in $0.5 \mathrm{ml}$ sodium acetate at $\mathrm{pH}$ 5.0, mixed with glass beads and water-equilibrated phenol, and incubated at $65^{\circ} \mathrm{C}$ with brief vortexing several times (Guthrie and Fink 1991). The supernatants were extracted two to three times with an equal volume of phenol/chloroform and the RNA precipitated with ethanol.

About 4-10 $\mu \mathrm{g}$ of RNA was separated by electrophoresis on denaturing gels of $10 \%$ polyacrylamide, $8 \mathrm{M}$ urea, or $1.2 \%$ formaldehyde agarose (Rose et al. 1990; Guthrie and Fink 1991). Northern assays were performed as described earlier (Balakin et al. 1994). The oligonucleotide probes were (1) 5'-CGATGGGTTCGTAAGCGTACTCCTACCGTGG $-3^{\prime}$ complementary to U14, (2) 5'-CGCTCAGATCTGCATGTGTTGTATAACACTGGC-3' complementary to snR190, and (3) 5'-CATAGGATGGGTCAAGATCATCGCGCC-3' complementary to U3. The primer extension termination assay was performed as described by Sigmund et al. (1988), with the following modification: 3 pmoles of $5^{\prime 32} \mathrm{P}$-labeled oligonucleotide was annealed with $2 \mu \mathrm{g}$ of total RNA with a gradual temperature change from $90^{\circ} \mathrm{C}$ to $25^{\circ} \mathrm{C}$ over $1 \mathrm{hr}$. The cDNA primer was $5^{\prime}$-GATAACTGATTTAATGAGCC-3'. 


\section{Acknowledgments}

We are glad to acknowledge earlier efforts to test the postulated U14:rRNA interaction made by Haodong $\mathrm{Li}_{\text {, Artur Jar- }}$ molowski, and Richard Lempicki of our laboratory. We thank R. Lempicki for preparing Figure 3, Yury Chernoff and Susan Liebman for advice on mutation of $18 \mathrm{~S}$ rDNA to hygromycin resistance, Jon Warner for yeast rDNA plasmids, Masayasu Nomura for plasmid pNOY199, and Elizabeth Furter-Graves for excellent editorial help. The research was supported by a grant from the National Institutes of Health (GM19351).

The publication costs of this article were defrayed in part by payment of page charges. This article must therefore be hereby marked "advertisement" in accordance with 18 USC section 1734 solely to indicate this fact.

\section{References}

Ausubel, F.M., R. Brent, R.E. Kingston, D.D. Moore, J.G. Seidman, J.A. Smith, and K. Struhl. 1989. Current protocols in molecular biology. John Wiley and Sons, Inc., New York.

Bachellerie, J-P., B. Michot, M. Nicoloso, A. Balakin, J. Ni, and M.J. Fournier. 1995. Antisense snoRNAs: A family of nucleolar RNAs with long complementarities to rRNA. Trends Biochem. Sci. 20: 261-264.

Balakin, A.G., R.A Lempicki, G.M. Huang, and M.J. Fournier. 1994. Saccharomyces cerevisiae Ul4 small nuclear RNA has little secondary structure and appears to be produced by post-transcriptional processing. /. Biol. Chem. 269: 739-746.

Baserga, S.J., W. Yang, and J.A. Steitz. 1991. An intact box C sequence in the U3 snRNA is required for binding of fibrillarin, the protein common to the major family of nucleolar snRNPs. EMBO I. 10: 2645-2651.

Beltrame, M. and D. Tollervey. 1992. Identification and functional analysis of two U3 binding sites on yeast pre-ribosomal RNA. EMBO /. 11: 1531-1542.

Beltrame, M., Y. Henry, and D. Tollervey. 1994. Mutational analysis of an essential binding site for the U3 snoRNA in the 5 ' external transcribed spacer of yeast pre-rRNA. Nucleic Acids Res. 22: 4057-4065.

Chernoff, Y.O., A. Vincent, and S.W. Liebman. 1994. Mutations in eukaryotic $18 \mathrm{~S}$ ribosomal RNA affect translational fidelity and resistance to aminoglycoside antibiotics. EMBO $/$. 13: 906-913.

Chu, S., R.H. Archer, J.M. Zengel, and L. Lindahl. 1994. The RNA of RNase MRP is required for normal processing of ribosomal RNA. Proc. Natl. Acad. Sci. 91: 659-663.

Dams, E., L. Hendriks, Y. Van de Peer, I.M. Neefs, G. Smits, I. Vandenbempt, and R. De Wachter. 1988. Compilation of small ribosomal subunit RNA sequences. Nucleic Acids Res. 16(S): r87-r173.

Eichler, D.C. and N. Craig. 1994. Processing of eukaryotic ribosomal RNA. Prog. Nucleic Acids Res. Mol. Biol. 49: 197239.

Elion, E.A. and J.R. Warner. 1984. The major promoter element of rRNA transcription in yeast lies $2 \mathrm{~kb}$ upstream. Cell 39: 663-673

Filipowicz, W. and T. Kiss. 1993. Structure and function of nucleolar snRNPs. Mol. Biol. Rep. 18: 149-156. (Published erratum appears in Mol. Biol. Rep. 1993. 18: 235).

Guthrie, C. and G.R. Fink. 1991. Guide to yeast genetics and molecular biology. Academic Press, San Diego, CA.

Henry, Y., H. Wood, J.P. Morrissey, E. Petfalski, S. Kearsey, and D. Tollervey. 1994. The $5^{\prime}$ end of yeast 5.8S rRNA is generated by exonuclease from an upstream cleavage site. $E M B O$ J. 13: 2452-2463.
Huang, G.M., A. Jarmolowski, J.C. Struck, and M.J. Fournier. 1992. Accumulation of U14 small nuclear RNA in Saccharomyces cerevisiae requires box $\mathrm{C}$, box $\mathrm{D}$, and a 5', 3' terminal stem. Mol. Cell. Biol. 12: 4456-4463.

Hughes, J.M. and M.J. Ares. 1991. Depletion of U3 small nucleolar RNA inhibits cleavage in the $5^{\prime}$ external transcribed spacer of yeast pre-ribosomal RNA and impairs formation of $18 \mathrm{~S}$ ribosomal RNA. EMBO /. 10: 4231-4239.

Jarmolowski, A., J. Zagorski, H.V. Li, and M.J. Fournier. 1990 Identification of essential elements in U14 RNA of Saccharomyces cerevisiae. EMBO I. 9: 4503-4509.

Kass, S., and B. Sollner-Webb. 1990. The first pre-rRNA processing event occurs in a large complex: Analysis by gel retardation, sedimentation, and UV cross-linking. Mol. Cell. Biol. 10: $4920-4931$.

Kass, S., K. Tyc, J.A. Steitz, and B. Sollner-Webb. 1990. The U3 small nucleolar ribonucleoprotein functions in the first step of preribosomal RNA processing. Cell 60: 897-908.

Kunkel, T.A. 1985. Rapid and efficient site-specific mutagenesis without phenotypic selection. Proc. Natl. Acad. Sci. 82: 488-492.

Leader, D.J., J.F. Sanders, R. Waugh, P. Shaw, and J.W.S. Brown. 1994. Molecular characterisation of plant U14 small nucleolar RNA genes: Closely linked genes are transcribed as polycistronic U14 transcripts. Nucleic Acids Res. 22: 5196 5203.

Li, H.V. 1991. "Structure-function analysis of the U14 small nuclear RNA of Saccharomyces cerevisiae." Ph.D. thesis, University of Massachusetts, Amherst, MA.

Li, H.V. and M.J. Fournier. 1992. U14 function in Saccharomyces cerevisiae can be provided by large deletion variants of yeast U14 and hybrid mouse-yeast U14 RNAs. EMBO $J$. 11: 683-689.

Li, H.V., J. Zagorski, and M.J. Fournier. 1990. Depletion of U14 small nuclear RNA (snR128) disrupts production of $18 \mathrm{~S}$ rRNA in Saccharomyces cerevisiae. Mol. Cell. Biol. 10: $1145-1152$.

Lygerou, Z., P. Mitchell, E. Petfalski, B. Seraphin, and D. Tollervey. 1994. The POP1 gene encodes a protein component common to the RNase MRP and RNase P ribonucleoproteins. Genes \& Dev. 8: 1423-1433.

Maser, R.L. and J.P. Calvet. 1989. U3 small nuclear RNA can be psoralen cross-linked in vivo to the $5^{\prime}$-external transcribed spacer of pre-ribosomal RNA. Proc. Natl. Acad. Sci. 86: 6523-6527.

Mattaj, I.W., D. Tollervey, and B. Seraphin. 1993. Small nuclear RNAs in messenger RNA and ribosomal RNA processing. FASEB I. 7: 47-53.

Maxwell, E.S. and M.J. Fournier. 1995. The small nucleolar RNAs. Annu. Rev. Biochem. 35: 897-934.

Morrissey, J.P. and D. Tollervey. 1993. Yeast snR30 is a small nucleolar RNA required for 18S rRNA synthesis. Mol. Cell. Biol. 13: 2469-2477.

. 1995. Birth of the snoRNPs: The evolution of RNase MRP and the eukaryotic pre-rRNA-processing system. Trends Biochem. Sci. 20: 78-82.

Mougey, E.B., L.K. Pape, and B. Sollner-Webb. 1993a. A U3 small nuclear ribonucleoprotein-requiring processing event in the $5^{\prime}$ external transcribed spacer of Xenopus precursor rRNA. Mol. Cell. Biol. 13: 5990-5998.

Mougey, E.B., M. O'Reilly, Y. Osheim, O.L. Miller Jr., A. Beyer, and B. Sollner-Webb. 1993b. The terminal balls characteristic of eukaryotic rRNA transcription units in chromatin spreads are rRNA processing complexes. Genes \& Dev. 7: 1609-1619.

Nogi, Y., Y. Ryoji, and M. Nomura. 1991. Synthesis of large 
rRNAs by RNA polymerase II in mutants of Saccharomyces cerevisiae defective in RNA polymerase I. Proc. Natl. Acad. Sci. 88: 3936-3966.

Petes, T.D. and D. Botstein. 1977. Simple Mendelian inheritance of the reiterated ribosomal DNA of yeast. Proc. Natl. Acad. Sci. 74: 5091-5095.

Raue, H.A. and R.J. Planta. 1995. The pathway to maturity: Processing of ribosomal RNA in Saccharomyces cerevisiae. Gene Expression (in press).

Raue, H.A., J. Klootwijk, and W. Musters. 1988. Evolutionary conservation of structure and function of high molecular weight ribosomal RNA. Prog. Biophys. Mol. Biol. 51: 77129.

Rose, M.D., F. Winston, and P. Hieter. 1990. Methods in yeast genetics. Cold Spring Harbor Laboratory Press, Cold Spring Harbor, New York.

Sambrook, J., E.F. Fritsch, and T. Maniatis. 1989. Molecular cloning: A laboratory manual, 2nd ed. Cold Spring Harbor Laboratory Press, Cold Spring Harbor, New York.

Schmitt, M.E. and D.A. Clayton. 1993. Nuclear RNase MRP is required for correct processing of pre-5.8S rRNA in Saccharomyces cerevisiae. Mol. Cell. Biol. 13: 7935-7941.

Sherman, F., G.R. Fink, and J.B. Hicks. 1985. Methods in yeast genetics. Cold Spring Harbor Laboratory, Cold Spring Harbor, New York.

Sigmund, C.D., M. Ettayebi, A. Borden, and E.A. Morgan. 1988. Antibiotic resistance mutations in ribosomal RNA genes of Escherichia coli. Methods Enzymol. 164: 673-690.

Spangler, E.A. and E.H. Blackburn. 1985. The nucleotide sequence of the $17 \mathrm{~S}$ ribosomal RNA gene of Tetrahymena thermophila and the identification of point mutations resulting in resistance to the antibiotics paromomycin and hygromycin. J. Biol. Chem. 260: 6334-6340.

Stroke, I. and A.M. Weiner. 1989. The $5^{\prime}$ end of U3 snRNA can be crosslinked in vivo to the external transcribed spacer of rat ribosomal RNA precursors. I. Mol. Biol. 210: 497-512.

Trinh-Rohlik, Q. and E.S. Maxwell. 1988. Homologous genes for mouse $4.5 \mathrm{~S}$ hybRNA are found in all eukaryotes and their low molecular weight RNA transcripts intermolecularly hybridize with eukaryotic $18 \mathrm{~S}$ ribosomal RNAs. Nucleic Acids Res. 16: 6041-6056.

Tyc, K. and J.A. Steitz. 1992. A new interaction between the mouse $5^{\prime}$ external transcribed spacer of pre-rRNA and U3 snRNA detected by psoralen crosslinking. Nucleic Acids Res. 20: 5375-5382.

Tycowski, K., M. Shu, and J.A. Steitz. 1994. Requirement for intron-encoded U22 small nucleolar RNA in $18 \mathrm{~S}$ ribosomal RNA maturation. Science 266: 1558-1561.

Woolford Jr., J.L. and J.R. Warner. 1991. The ribosome and its synthesis. In The molecular and cellular biology of the yeast Saccharomyces (ed. J.R. Broach, J.R. Pringle, and E.W. Jones), Vol. 1. pp. 587-626. Cold Spring Harbor Laboratory Press, Cold Spring Harbor, New York. 


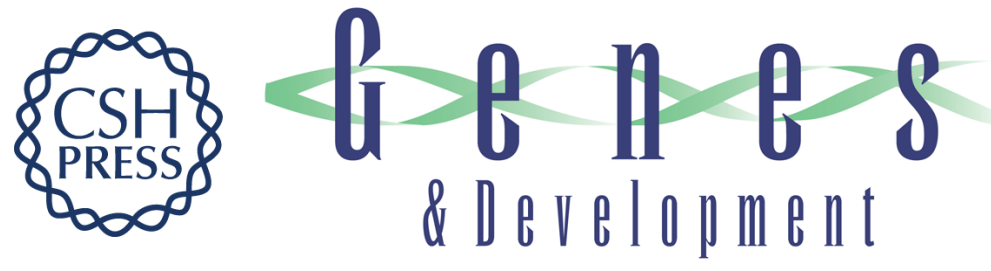

\section{U14 base-pairs with 18S rRNA: a novel snoRNA interaction required for rRNA processing.}

W Q Liang and M J Fournier

Genes Dev. 1995, 9:

Access the most recent version at doi:10.1101/gad.9.19.2433

References This article cites 39 articles, 15 of which can be accessed free at:

http://genesdev.cshlp.org/content/9/19/2433.full.html\#ref-list-1

License

Email Alerting

Service

Receive free email alerts when new articles cite this article - sign up in the box at the top right corner of the article or click here.

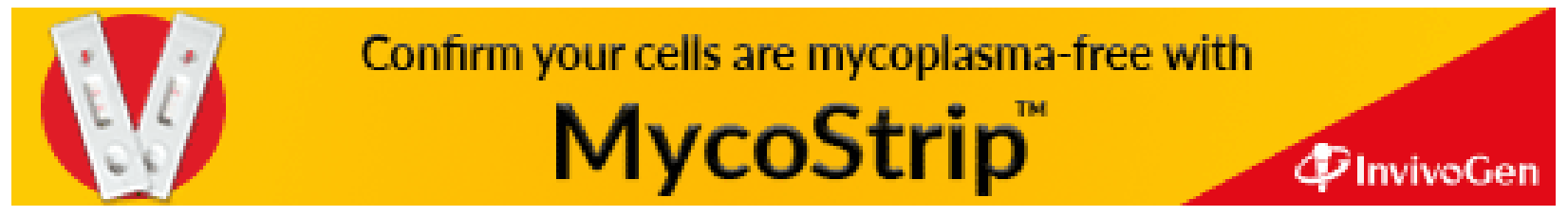

